\title{
Towards Reggeon Field Theory in QCD
}

\author{
Michael Lublinsky
}

University of Connecticut

In collaboration with Alex Kovner 


\section{RFT - History}

60 V. Gribov: RFT with supercritical bare Pomeron $s^{\Delta}, \Delta>0$.

$$
\mathrm{QCD}(s \rightarrow \infty) \longrightarrow \mathrm{RFT} \longrightarrow \text { Confinement }
$$

$\longrightarrow \quad$ String Theory

70‘ BFKL (Balitsky, Fadin, Kuraev, Lipatov) ladder - Hard Pomeron, $s^{c \alpha s}$

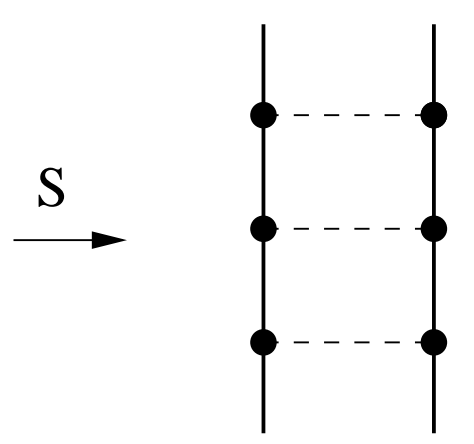

BFKL

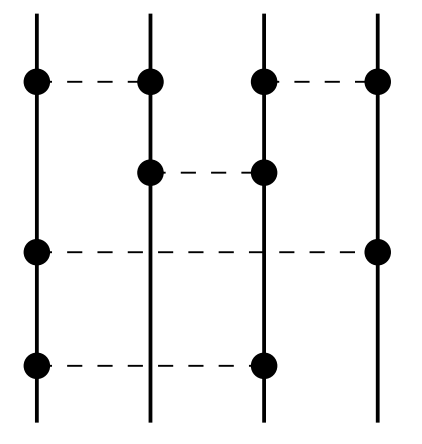

BKP

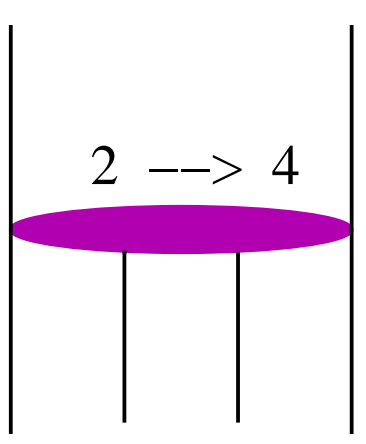

3P Vertex

80‘ BKP (Bartels, Kwiecinski, Praszalowicz), GLR (L. Gribov, Levin, Ryskin)

90، 3P Vertex (Bartels, Wusthoff, M. Braun), Lipatov's action, Mueller's dipole model, B-JIMWLK (Balitsky, Jalilian Marian, Iancu, McLerran, Leonidov, Kovner).

2005 JIMWLK+, KLWMIJ+, Dense-Dilute Duality (DDD), Self-Duality of RFT. 


\section{High Energy Scattering}

HERA - eRHIC
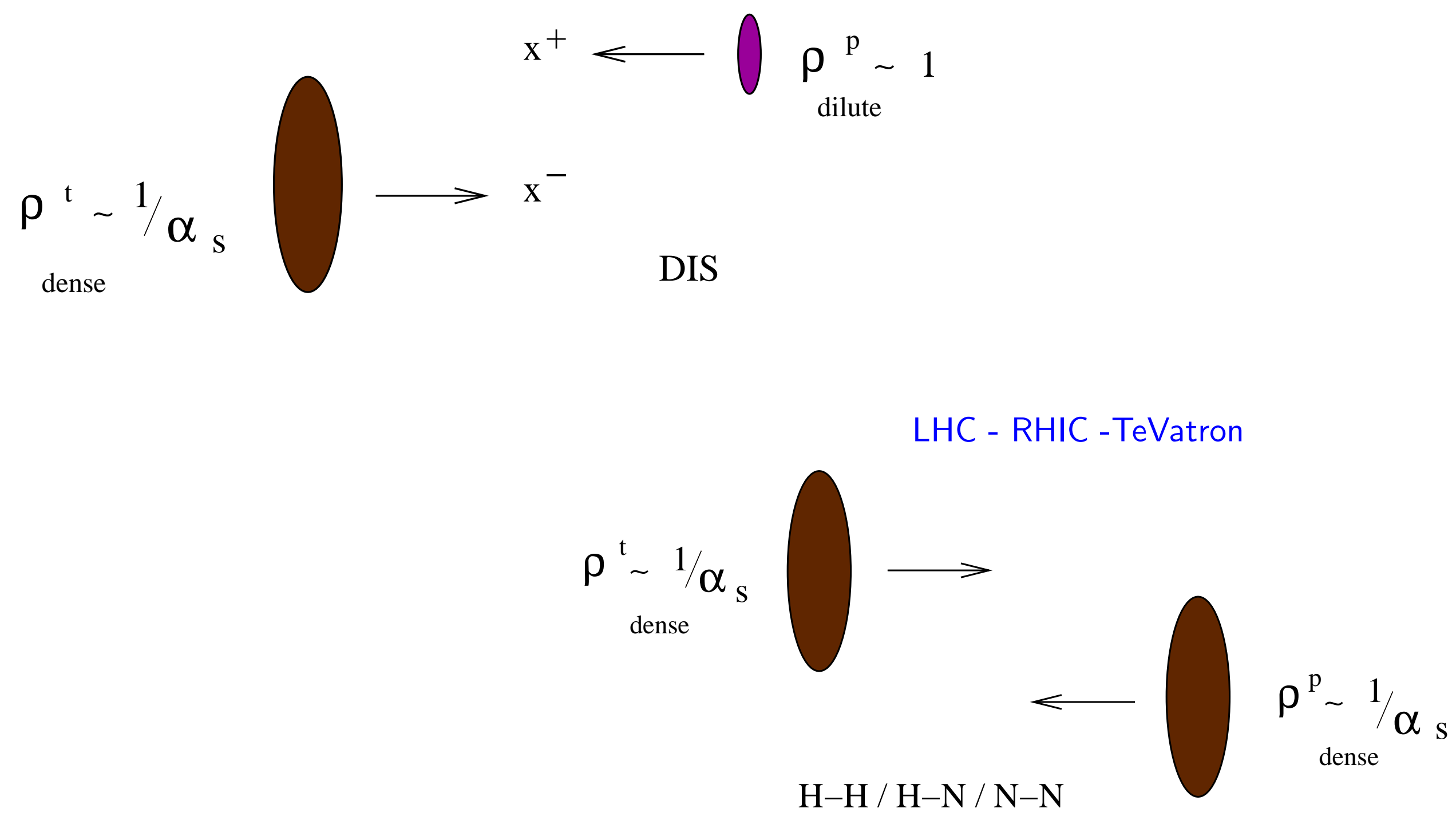


$$
\begin{array}{ccc}
\text { Target }\left(\rho^{t}=\rho^{+} ; k^{+}>\Lambda\right) & \text { Projectile }\left(\rho^{p}=\rho^{-} ; k^{-}>\Lambda\right) \\
\langle T| & \rightarrow & \leftarrow|P\rangle
\end{array}
$$

$S$-matrix:

$$
S(Y)=\left\langle T\left\langle P\left|\hat{s}\left(\rho^{t}, \rho^{p}\right)\right| P\right\rangle T\right\rangle
$$

Projectile averaged $s$-matrix:

$$
\begin{gathered}
\Sigma_{Y-Y_{0}}^{p}\left(\rho^{t}\right)=\left\langle P\left|\hat{s}\left(\rho^{t}, \rho^{p}\right)\right| P\right\rangle=\int D \rho^{p} \hat{s}\left(\rho^{t}, \rho^{p}\right) W_{Y-Y_{0}}^{p}\left[\rho^{p}\right] \\
S(Y)=\int D \rho^{t} \Sigma_{Y-Y_{0}}^{p}\left[\rho^{t}\right] W_{Y_{0}}^{t}\left[\rho^{t}\right]
\end{gathered}
$$


Boosting projectile

$$
|P\rangle_{Y} \rightarrow|P\rangle_{Y+\delta Y}
$$

$$
\frac{d \Sigma^{p}}{d Y}=H_{\Sigma}^{H E} \Sigma^{p}
$$

Boosting target

$$
\begin{aligned}
|T\rangle_{Y} & \rightarrow|T\rangle_{Y+\delta Y} \\
& \frac{d W^{t}}{d Y}=H_{W}^{H E} W^{t}
\end{aligned}
$$

Lorentz invariance:

$$
d S / d Y_{0}=0 \quad \rightarrow \quad H_{\Sigma}^{H E}=H_{W}^{H E}
$$

$S$-matrix evolution:

$$
H^{R F T} \rightarrow \text { the RFT Hamiltonian }
$$

$$
\frac{d S}{d Y}=\int D \rho^{t} \quad \Sigma_{Y-Y_{0}}^{p}\left[\rho^{t}\right] \quad H^{R F T}\left[\rho^{t}, \delta / \delta \rho^{t}\right] \quad W_{Y_{0}}^{t}\left[\rho^{t}\right]
$$




\section{Dense/Dilute limit}

$H^{K L W M I J}=H^{R F T}(\rho \rightarrow 0) ; \quad H^{J I M W L K}=H^{R F T}(\rho \rightarrow \infty)$

J I MWLK - Jalilian Marian, lancu, McLerran, Leonidov, Kovner (1997-2002)

KLW M I J - A. Kovner and M.L., Phys.Rev.D71:085004, 2005

Evolution with Pomeron Loops (model):

$$
H^{R F T} \simeq H^{J I M W L K}(\rho \rightarrow \infty) " \prime+" H^{K L W M I J}(\rho \rightarrow 0)
$$




\section{KLWMIJ Hamiltonian (dilute limit)}

$$
\langle\mathcal{O}\rangle_{Y} \equiv\left\langle P\left|\hat{\mathcal{O}}\left[\rho^{p}\right]\right| P\right\rangle_{Y}=\int D \rho^{p} \mathcal{O}\left[\rho^{p}\right] W_{Y}^{p}\left[\rho^{p}\right]
$$

$$
\frac{d\langle\mathcal{O}\rangle_{Y}}{d Y}=\int D \rho^{p} \quad \mathcal{O}\left[\rho^{p}\right] H^{K L W M I J} W_{Y}^{p}\left[\rho^{p}\right]
$$

Boosting projectile $\quad|P\rangle_{Y} \rightarrow|P\rangle_{Y+\delta Y}$

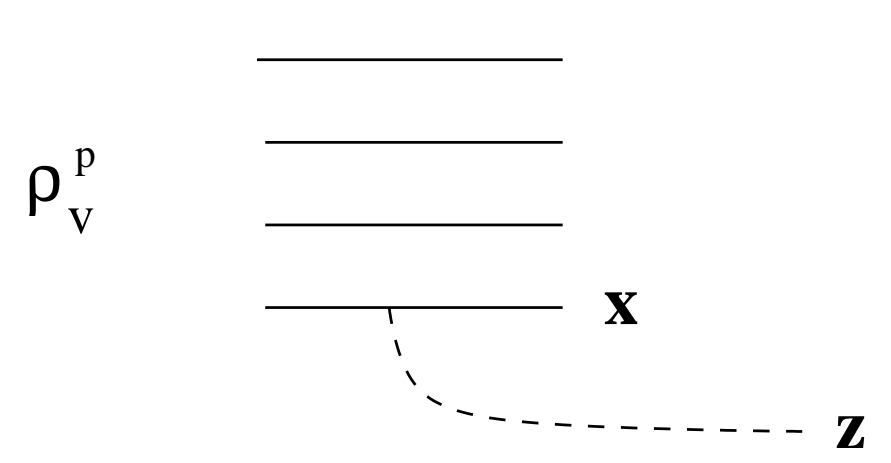

Linear evolution means $\delta \rho \propto \rho_{v}^{p}$

Emission amplitude is given by the Weizsaker-Williams field

$b_{i}^{a}(z)=\frac{g}{2 \pi} \int d^{2} x \frac{(x-z)_{i}}{(x-z)^{2}} \rho^{p a}(x)$ 


$$
\rho^{p} \longrightarrow \rho^{p}+T^{a} ; \quad \hat{\mathcal{O}}\left[\rho^{p}\right] \longrightarrow \hat{\mathcal{O}}\left[\rho^{p}+T^{a}\right]
$$

Dual Wilson line

$$
R(z)=P \exp \left\{\int d x^{-} T^{a} \frac{\delta}{\delta \rho^{a}\left(z, x^{-}\right)}\right\} \quad R(z) \rho^{a}(z)=\rho^{a}(z)+T^{a}
$$

$$
\begin{aligned}
& \begin{array}{llll}
\delta & \delta & \delta & \delta
\end{array} \\
& H^{K L W M I J}[\rho, \delta / \delta \rho]=\int_{z} b_{i}^{a}(z)[1-R(z)]^{a b} b_{i}^{b}(z) \\
& \delta \rho, \delta \rho \quad \delta \rho \quad \delta \rho \\
& \rho \text { KLWMIJ ' } \rho
\end{aligned}
$$




\section{JIMWLK Hamiltonian (dense limit)}

$$
H^{J I M W L K}\left[\alpha^{t}, \frac{\delta}{\delta \alpha^{t}}\right]=\int_{z} b_{i}\left[\frac{\delta}{\delta \alpha^{t}}\right][1-S(z)] b_{i}\left[\frac{\delta}{\delta \alpha^{t}}\right]
$$

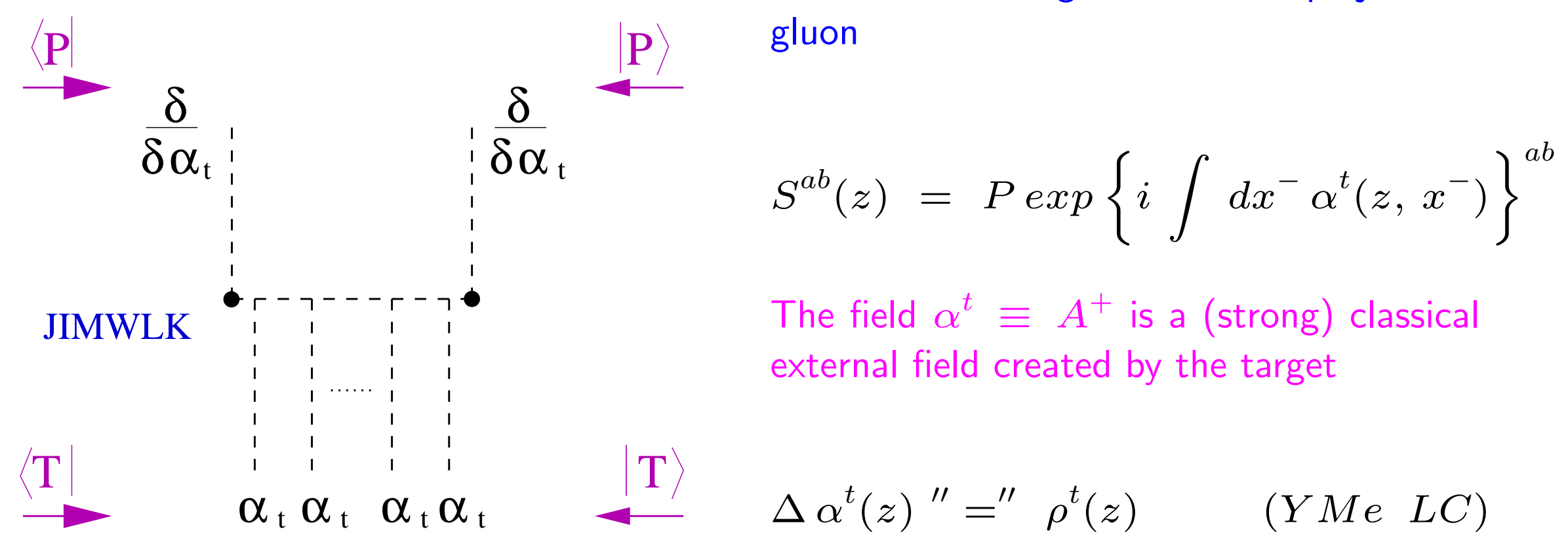




\section{Beyond JIMWLK: JIMWLK+}

A. Kovner and M.L., JHEP 0503:001,2005

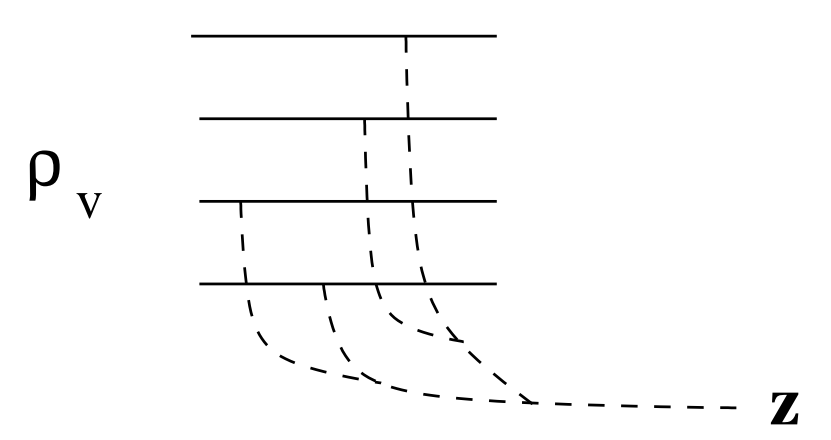

$H^{J I M W L K+}=\int_{z} b_{i}[1-S(z)] b_{i}$
Coherent emission of a single gluon

$D_{i}[b] b_{i}^{a}=\rho_{p}^{a}$

$b$ is non-linear in $\rho^{p}=\rho_{v} \geq 1$

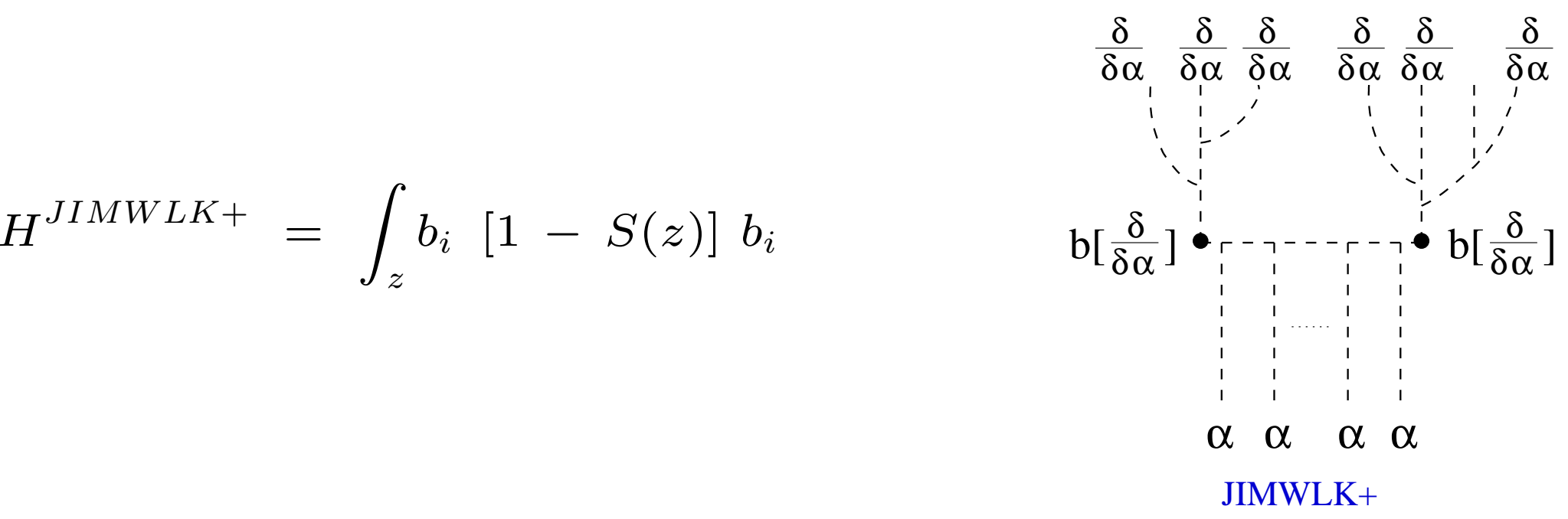

Y. Hatta, E. lancu, L. McLerran, A. Stasto and D.N. Triantafyllopoulos, Nucl.Phys. A764: 423,2006;

I. Balitsky, Phys.Rev. D72:074027,2005 


\section{DDD - Dense Dilute Duality}

$H^{J I M W L K}(\rho \rightarrow \infty)=\alpha_{s} \int_{x, y, z} \frac{(z-x)_{i}(z-y)_{i}}{(z-x)^{2}(z-y)^{2}} \frac{\delta}{\delta \alpha^{a}(x)}[1-S(z)]^{a b} \frac{\delta}{\delta \alpha^{b}(y)}$
$H^{K L W M I J}(\rho \rightarrow 0)=\alpha_{s} \int_{x, y, z} \frac{(z-x)_{i}(z-y)_{i}}{(z-x)^{2}(z-y)^{2}} \rho^{a}(x)[1-R(z)]^{a b} \rho^{b}(y)$

DDD transformation:

$$
i \alpha \rightarrow \frac{\delta}{\delta \rho} ; \quad \frac{\delta}{\delta \alpha} \rightarrow i \rho \quad S \rightarrow R
$$

$$
H^{J I M W L K} \leftrightarrow H^{K L W M I J}
$$




\section{Self-Duality of High Energy Evolution}

A. Kovner and M.L. (PRL 94:181603,2005)

- Lorentz Invariance (LI)

- Eikonal Approximation (EA)

- Projectile - Target Democracy (PTD)

$$
H^{R F T}(i \alpha, \delta / \delta \alpha)=H^{R F T}(\delta / \delta \rho, i \rho)
$$

Self-Duality $=t$-channel unitarity? 


\section{Reggeon Field Theory in QCD}

A. Kovner and M.L. (hep-ph/0512316, hep-ph/0604085)

- Hamiltonian $(2+1)$ dimensional interacting non-local field theory.

- The basic "quantum Reggeon field" is the unitary matrix $R(S)$.

- Symmetry: DDD

- Two zero energy degenerate vacua ("Yang" and "Yin"), DDD is spontaneously broken.

- Spectrum of excitations is twice degenerate

(gluons and "holes")

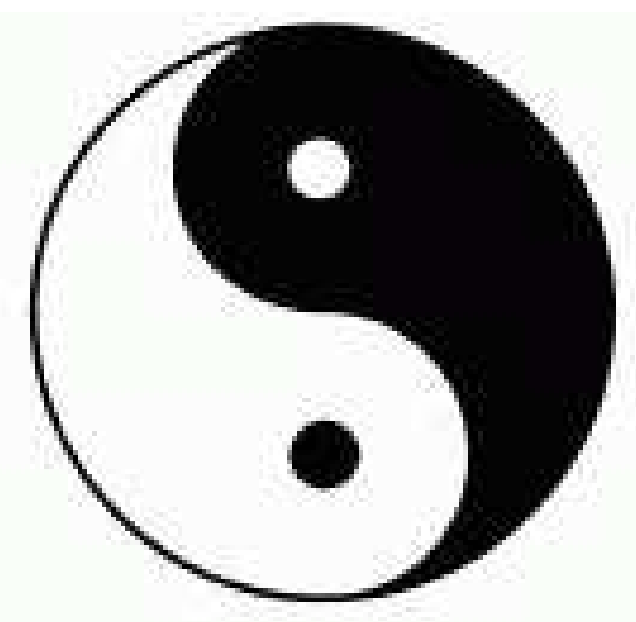

- More symmetries: $S U_{V}(N), Z_{2} ; 2$-d Conformal invariance?

- BFKL Pomeron is a tachyon 


\section{Summary and Outlook}

- Some progress has been achieved in understanding high energy limit of QCD: JIMWLK+, KLWMIJ+

DDD, Self-Duality

- We are in a quest for a complete QCD - RFT.

- Self-Duality

- $s$ - and $t$ - channel unitarity

- Symmetries? Conformal Symmetry? Integrability?

- Phenomenology (LHC, HERA, RHIC, TeVatron) 